\title{
SEARCH FOR STABLE ORBITS AROUND THE BINARY ASTEROID SYSTEMS 1999 KW4 AND DIDYMOS
}

\author{
Thais C. Oliveira and Antonio F. B. A. Prado \\ Space Mechanic and Tecnology, National Institute for Space Research/INPE, Sao Jose dos Camopo, SP, \\ Brazil.
}

Received July 4 2019; accepted January 29 2020

\begin{abstract}
This work includes analytical and numerical studies of spacecrafts orbiting two binary asteroid systems: $1999 \mathrm{KW} 4$ and Didymos. The binary systems are modeled as full irregular bodies, such that the whole evolution of the results will show the impact of the irregular gravity field in the lifetime and dynamics of the spacecraft's orbit. The equations of motion of the binary system and the spacecraft are derived from Lagrange Equations. The solar radiation pressure is consired in the dynamics of the spacecraft.Two distinct methods are used to search for stable orbits around the binary systems. One is called the grid search method, which defines the main body as a point mass to estimate the initial state of the spacecraft based on a circular Keplerian orbit. The second method is the search for periodic orbits based on zero-velocity surfaces.
\end{abstract}

\section{RESUMEN}

Este trabajo presenta estudios analíticos y numéricos sobre naves espaciales en órbita en torno a dos asteroides binarios: 1999 KW4 y Didymos. Se modelan los sistemas binarios como cuerpos irregulares, de modo que la evolución muestre el impacto del campo de gravedad irregular sobre los tiempos de vida y la dinámica de la órbita de la nave. Se derivan las ecuaciones de movimiento del sistema binario y de la nave a partir de las ecuaciones de Lagrange. Se considera la presión de radiación solar. Se buscan órbitas estables en torno a los sistemas binarios mediante dos métodos. El primero se conoce como el método de búsqueda en la malla, y considera al cuerpo principal como punto masa para estimar el estado inicial de la nave a partir de una órbita Kepleriana circular. El segundo busca órbitas periódicas mediante las superficies de cero velocidad.

Key Words: methods: numerical — minor planets, asteroids: general — space vehicles

\section{INTRODUCTION}

There is a great scientific interest in asteroids due to their relatively unchanged status as remnant debris from the solar system formation process, around 4.6 billion years ago.

Asteroids are leftovers from the Solar System that can offer clues to the chemical mixture from which planets were formed. In addition, asteroids can pose a danger to Earth depending on the size and course of the object. Therefore, gaining a better understanding of the physical and chemical parameters of the asteroids can help humans to prevent impacts between these objects and the Earth, e.g. by using a gravity tractor, a nuclear explosion, a conventional rocket engine, or a solar energy engine. Moreover, some asteroids have the potential of being used for mining.

The Solar System has different groups of asteroids. The near-Earth asteroids (NEAs) group includes asteroids in the Earth's neighborhood and that have a perihelion distance less than 1.3 AU (NASA 2019). Over 
20,000 NEAs have been discovered, of which more than 895 are large asteroids with an estimated diameter greater than $1 \mathrm{~km}$ while over 8,600 NEAs have an estimated diameter between $140 \mathrm{~m}$ to $1 \mathrm{~km}$ (JPL 2019).

The group with the largest population of asteroids is the main-belt group. The main-belt asteroids orbit the Sun and lie between Mars and Jupiter. The belt is estimated to contain between 1.1 and 1.9 million asteroids larger than $1 \mathrm{~km}$ in diameter, and millions of smaller ones (NASA 2019).

The last group of asteroids is the Trojan. The Trojans share an orbit with a larger planet. They are located at the Lagrangian points $L_{4}$ and $L_{5}$. The Jupiter Trojans form the most significant population of Trojan asteroids. It is thought that they are as numerous as those of the main-asteroid belt (NASA 2019).

There are some asteroids that have natural satellites. The first one was discovered in 1993 around 243 Ida. Since then, many other binary systems have been discovered. It is estimated that there are more than 160 binary asteroids systems: around 15\% are NEAs, 2 to $3 \%$ are in the main-belt and between 10 to $30 \%$ belong to other groups of trans-Neptunian objects (Johnston 2014; Margot et al. 2015). Therefore, it is not surprising that missions to binary asteroid systems will occur in the future.

The Asteroid Impact and Deflection Assessment (AIDA) mission is a proposed pair of space probes which would study and demonstrate the kinetic effects of crashing an impactor spacecraft into the secondary body of a binary asteroid system. Its target would be the moonlet of the binary near-Earth asteroid (65803) Didymos, which is one of the two binary asteroid systems studied in this paper.

The second binary asteroid system studied in this paper is 1999 KW4. This primary asteroid was discovered by LINEAR on May 20, 1999. Its companion was discovered 2001 May 21 using radar and light curve observations (Ostro et al. 2006). $1999 \mathrm{KW} 4$ has the least accessible $\Delta V$ for a spacecraft mission of any known binary near-Earth asteroid due to its heliocentric inclination of 39 degrees, eccentricity of 0.7 , and semi-major axis of 0.64 au (astronomical units). 1999 KW4 has been classified as a "Potentially Hazardous Asteroid" by the Minor Planet Center (JPL 2019).

The search for stable orbits around a binary system is performed by utilizing theoretical results and numerical methods that have been used in the circular restricted three-body problem (Scheeres et al. 1996; Woo 2014; Yu \& Baoyin 2012a; Yu \& Baoyin 2012b).

Among the many research topics in the circular restricted three-body problem, the periodic orbit is a fundamental and important part. Generally, there are five kinds of periodic orbits in the global spatial space: libration point orbits, orbits about the primary, orbits about the secondary, orbits about the whole binary system, and other orbits about neither asteroid (Shi et al. 2018a).

The study of periodic orbits provides a general understanding of the stability and formation of natural orbits; stable orbits can be directly adopted for close-proximity operations in space (Hu \& Scheeres 2004). Particularly, in the study of asteroids, periodic orbits indicate the potential locations of mutual satellites, which is significant for assessing the spatial environment (Yu \& Baoyin 2012b).

Periodic orbits around asteroids have been approximated using models with various degrees of sophistication. In general, one will make some assumptions, such as using a simple shape model and restricting the binary system to be synchronous. Then, the dynamical substitutes in a more precise model can be further determined based on the prior calculated results (Chappaz \& Howell 2015; Hou \& Xin 2018). To describe the non-spherical shape of an asteroid, an ellipsoid can be used to represent it, which generates the sphere-ellipsoid model and the ellipsoid-ellipsoid model for the binary asteroid systems. Bellerose \& Scheeres $(2008,2008)$ studied the periodic orbits in the sphere-ellipsoid binary system, especially the libration point orbits. Both the sphere-ellipsoid binary system and the ellipsoid-ellipsoid binary system were investigated by Chappaz \& Howell (2015), where the resonant orbits were calculated and analyzed in addition to the libration point orbits. Shang et al. (2015) searched the global periodic orbits in the ellipsoid-ellipsoid binary system based on the symmetry of the orbits and obtained different families of periodic orbits. Their results showed that the periodic orbits in an ellipsoidellipsoid binary system are analogous to those in the circular restricted three-body problem. The same results are obtained in this paper.

A crucial difference is that the ellipsoid model shows symmetry while the real asteroid does not. The highfidelity models, such as the polyhedron model and the spherical harmonics model, can reflect the asymmetry of the real binary system. Particularly, the polyhedron model derived by Scheers (1996) is suitable near the surface of the asteroid. Libration point orbits of Mars-Phobos binary system were investigated by Biggs (2015) using spherical harmonics. They indicated that the irregular shape has a significant influence and that the connections between families of periodic orbits are different from those in the circular restricted three-body 

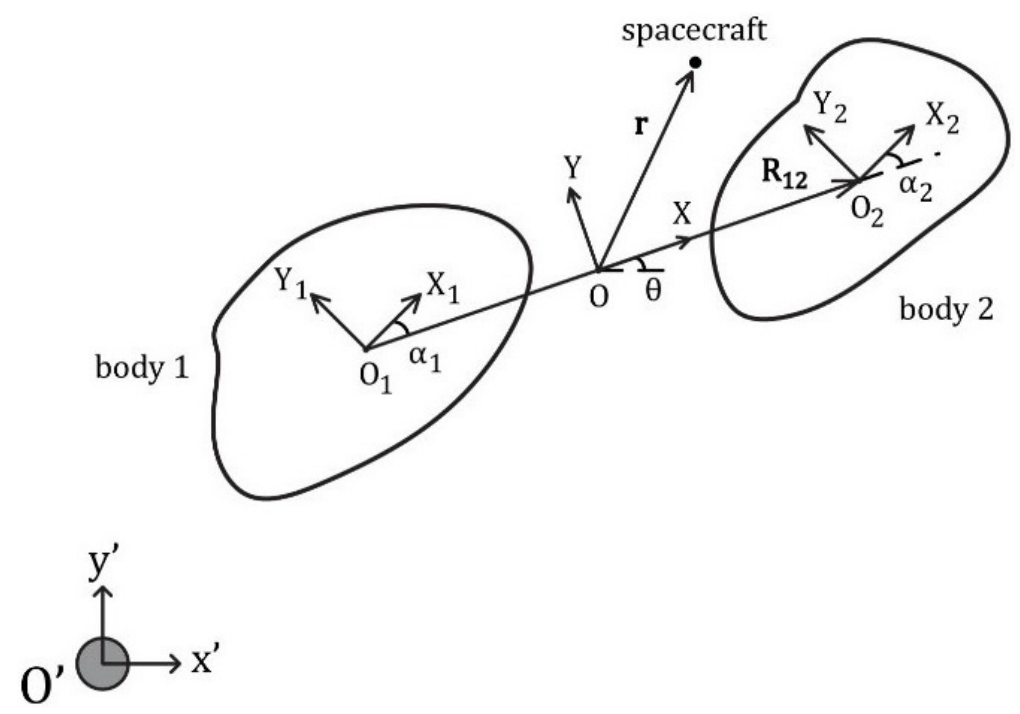

Fig. 1. Illustration of the binary system (Woo \& Misra 2013).

problem. Based on the polyhedron model, Scheeres et al. (2017) also revealed differences of the libration point orbits and investigated the retrograde periodic orbits about the Phobos. Shi et al. (2018b) studied the libration point orbits near the binary asteroid system 1999 KW4 using a polyhedron-polyhedron model.

This paper considers the dynamics of the 1999 KW4 and Didymos binary systems as full irregular bodies, from ellipsoids to double-truncated cones. This paper focuses on the search for stable orbits for spacecrafts around these systems based on two distinct methods: the grid search method and zero velocity curves. The grid search method defines the main body as a point mass to estimate the initial state of the spacecraft based on a Keplerian orbit. The second method searches for periodic orbits based on zero-velocity surfaces. All the simulations consider the solar radiation pressure as a disturbing force on the dynamics of the spacecraft. The solar radiation force is a non-conservative force and it can play a key role in the spacecraft orbits around the Solar System. The solar radiation pressure originates from the interaction of the satellite's surface with the photons emitted by the Sun. It is assumed that each photon that hits the satellite is either absorbed or reflected in a specular or diffuse way.

Periodic orbits are important keys to understand the motion of a massless particle in the vicinity of a binary asteroid system. Due to the complex gravity generated by the irregular-shaped asteroids, it is difficult to generate periodic orbits with an analytical method, except with linearized dynamics in a small region around the libration points. This paper presents a numerical method to search for planar periodic orbits in a global space. The search method can also be used to find periodic orbits in the vicinity of other binary systems.

\section{MATHEMATICAL FORMULATION}

This paper studies the planar motion of a binary asteroid system, where a full two body problem is considered to account for the mutual motion of the asteroids. The two arbitrarily shaped asteroids have masses $m_{1}$ and $m_{2}$, as shown in Figure 1. The system $O^{\prime} \mathrm{x}^{\prime} \mathrm{y}^{\prime} \mathrm{z}^{\prime}$ is an inertial reference frame with the unit vectors $\mathbf{i}^{\prime}, \mathbf{j}^{\prime}$ and $\mathbf{k}^{\prime}$.

The center of mass of the binary system is located at $\mathrm{O}$, which is also the origin of the local system OXYZ. The unit vectors of the coordinate system OXYZ are given by $\mathbf{i}, \mathbf{j}$ and $\mathbf{k}$. Note that this reference frame is a synodic one.

The centers of mass of $m_{1}$ and $m_{2}$ are given by $O_{1}$ and $O_{2}$, respectively. The vector $\mathbf{R}_{12}$ connects the point $O_{1}$ to $O_{2}$. The direction of $\mathbf{R}_{12}$ is the positive $\mathrm{X}$ axis orientation of the right-handed coordinate system OXYZ. The orientation of OXYZ relative to the inertial frame is given by the angle $\theta$ (Woo \& Misra 2014).

Each asteroid has a body-fixed frame, given by $O_{i} X_{i} Y_{i} Z_{i}$, for $i=1,2$. The orientation of the body $i$ with respect to the local frame is given by the angle $\alpha_{i}$. The unit vectors of the body-fixed frame $i$ is given by $\mathbf{i}_{\mathbf{i}}, \mathbf{j}_{\mathbf{i}}$ and $\mathbf{k}_{\mathbf{i}}$. 
The mass of the spacecraft is negligible for the system dynamics and the motion of the spacecraft is given by the vector $\mathbf{r}=x \mathbf{i}+y \mathbf{j}+z \mathbf{k}$ at the local reference frame OXYZ.

\subsection{Dynamics of the System}

\subsubsection{Lagrange's Mathematical Formulation}

The equations of motion of the asteroid binary system are derived from the Lagrange equations. The generalized coordinates are the distance $R_{12}$, the orientation angle $\theta$ and the orientation body angles $\alpha_{1}$ and $\alpha_{2}$.

The kinetic energy for the planar binary system based on Figure 1 is given by (Woo \& Misra 2013):

$$
T=\frac{1}{2}\left(m_{1}+m_{2}\right) V_{0}^{2}+\frac{1}{2} \frac{m_{1} m_{2}}{m_{1}+m_{2}}\left(\dot{R}_{12}^{2}+R_{12}^{2} \dot{\theta}^{2}\right)+\frac{1}{2} I_{z z 1}\left(\dot{\theta}+\dot{\alpha}_{1}\right)^{2}+\frac{1}{2} I_{z z 2}\left(\dot{\theta}+\dot{\alpha_{2}}\right)^{2},
$$

where $V_{0}=\left(\dot{\mathbf{R}}_{\mathbf{0}} \cdot \dot{\mathbf{R}}_{\mathbf{0}}\right)^{1 / 2}$ is the speed of the center of mass in the inertial frame and $\mathbf{R}_{\mathbf{0}}$ is the position vector of $\mathrm{O}$ in the inertial system; finally, $I_{x x i}, I_{y y i}$ and $I_{z z i}$ are the moments of inertia of the $i^{\text {th }}$ body in the $O X_{i} Y_{i} Z_{i}$ coordinate system, for $i=1,2$.

The potential energy is given by (Woo \& Misra 2013):

$$
\begin{aligned}
U= & -\frac{G m_{0}\left(m_{1}+m_{2}\right)}{R_{0}}-\frac{G m_{1} m_{2}}{R_{12}}+\frac{3 G m_{2}}{2 R_{12}^{3}}\left[I_{x x 1} \cos ^{2} \alpha_{1}+I_{y y 1} \sin ^{2} \alpha_{1}-\frac{1}{3}\left(I_{x x 1}+I_{y y 1}+I_{z z 1}\right)\right]+ \\
& \frac{3 G m_{1}}{2 R_{12}^{3}}\left[I_{x x 2} \cos ^{2} \alpha_{2}+I_{y y 2} \sin ^{2} \alpha_{2}-\frac{1}{3}\left(I_{x x 2}+I_{y y 2}+I_{z z 2}\right)\right],
\end{aligned}
$$

where $m_{0}$ is the mass of the Sun.

Assuming no other external forces, the Lagrange equations are given by:

$$
\frac{d}{d t} \frac{\partial T}{\partial \dot{q}_{j}}-\frac{\partial T}{\partial q_{j}}+\frac{\partial U}{\partial q_{j}}=0
$$

where $q_{j}=R_{12}, \theta, \alpha_{1}$ and $\alpha_{2}$.

\subsubsection{Non-Dimensionalization and other Variables Definitions}

We will use dimensionless variables to study the system. To this end, we introduce the mass ratio $v=\frac{m_{1}}{m_{1}+m_{2}}$, the characteristic length of the bodies $r_{0}$ and the characteristic length of the mutual bodies $L$.

The moments of inertia $I_{x x}, I_{y y}$ and $I_{z z}$ of each body are described by their non-dimensional radii of gyration $p_{x x}, p_{y y}$ and $p_{z z}$. The radii of gyration are given by $p_{x x i}=\sqrt{\frac{I_{x x i}}{m_{i} r_{0}^{2}}}, p_{y y i}=\sqrt{\frac{I_{y y i}}{m_{i} r_{0}^{2}}}$ and $p_{z z i}=\sqrt{\frac{I_{z z i}}{m_{i} r_{0}^{2}}}$, for $i=1,2$ Woo \& Misra $(2013,2014)$.

The non-dimensionalized position vector of the spacecraft is given by:

$$
\overline{\mathbf{r}}=\frac{\mathbf{r}}{L}=\frac{x}{L} \mathbf{i}+\frac{y}{L} \mathbf{j}+\frac{z}{L} \mathbf{k} .
$$

The non-dimensionalized time is defined by: $\tau=n t$, where $n$ is the mean motion of a circular orbit with radius $L$. The mean motion is given as follows: (Bellerose \& Scheeres 2008; Woo \& Misra 2014)

$$
n=\sqrt{G\left(m_{1}+m_{2}\right) / L^{3}} .
$$

The time derivatives can be written as:

$$
\frac{d(\cdot)}{d t}=n \frac{d(\cdot)}{d \tau}=n(\cdot)^{\prime}
$$

The distance $R_{12}$ is replaced by the variable: $u=L / R_{12}$. 


\subsection{The Circular Mutual Orbit Between the Binary Asteroid System}

The equations of motion derived in equation 3 in terms of the non-dimensional quantities are given by (Woo \& Misra 2013):

$$
\begin{gathered}
u^{\prime \prime}-\frac{2 u^{\prime 2}}{u}+u \theta^{\prime 2}-u^{4}-\epsilon \frac{3}{4} u^{6}\left(k_{12}^{2}+3 k_{1}^{2} \cos 2 \alpha_{1}+3 k_{2}^{2} \cos 2 \alpha_{2}\right)=0 \\
\frac{d}{d t}\left(\theta^{\prime}+\epsilon u^{2}\left[\frac{p_{z z 1}^{2}}{1-v}\left(\theta^{\prime}+\alpha_{1}^{\prime}\right)+\frac{p_{z z 2}^{2}}{v}\left(\theta^{\prime}+\alpha_{2}^{\prime}\right)\right]\right)=0 \\
\theta^{\prime \prime}+\alpha_{1}^{\prime \prime}+\frac{3}{2}(1-v) \frac{k_{1}^{2}}{p_{z z 1}^{2}} u^{3} \sin \left(2 \alpha_{1}\right)=0 \\
\theta^{\prime \prime}+\alpha_{2}^{\prime \prime}+\frac{3}{2} v \frac{k_{2}^{2}}{p_{z z 2}^{2}} u^{3} \sin \left(2 \alpha_{2}\right)=0
\end{gathered}
$$

where $\epsilon=\left[\frac{r_{0}}{L}\right]^{2} ; \quad k_{12}^{2}=2 p_{z z 1}^{2}-p_{x x 1}^{2}-p_{y y 1}^{2}+2 p_{z z 2}^{2}-p_{x x 2}^{2}-p_{y y 2}^{2} ; \quad k_{1}^{2}=p_{y y 1}^{2}-p_{x x 1}^{2} ; \quad$ and $k_{2}^{2}=p_{y y 2}^{2}-p_{x x 2}^{2}$.

This paper considers binary systems in a mutual circular orbit. This means that the system is in a relative equilibrium, where $u, \theta^{\prime}, \alpha_{1}$ and $\alpha_{2}$ are constant. In other words, $u^{\prime \prime}(\tau)=u^{\prime}(\tau)=\theta^{\prime \prime}(\tau)=\alpha_{1}^{\prime \prime}(\tau)=\alpha_{2}^{\prime \prime}(\tau)=0$.

It is assumed that $\alpha_{1}(\tau=0)=\alpha_{2}(\tau=0)=0$ for all simulations and also that the body-fixed frames centered at $O_{1}$ and $O_{2}$ are chosen to be aligned with the principal axes. The initial condition for the planar binary system to move in a circular orbit taking $\alpha_{1}=\alpha_{2}=0$ is given as follows (Woo 2014; Woo \& Misra 2013):

$$
\theta^{\prime}=H\left[1+\epsilon u^{2}\left(\frac{p_{z z 1}^{2}}{1-v}+\frac{p_{z z 2}^{2}}{v}\right)\right]^{-1},
$$

where $H$ is the constant of integration of equation 8 .

\subsection{The Equation of Motion of the Spacecraft}

The orbital motion of the spacecraft is studied by considering the restricted full three-body problem. The term "restricted" indicates that the mass of the spacecraft does not affect the dynamics of the binary asteroid bodies. Assuming that the binary asteroid system orbits in a circular orbit, the equations of motion of the spacecraft in the OXYZ reference frame are given by (Woo \& Misra 2015):

$$
\begin{gathered}
x^{\prime \prime}-2 \theta^{\prime} y^{\prime}-\theta^{2} x=f_{1}(x, y, z), \\
y^{\prime \prime}+2 \theta^{\prime} x^{\prime}-\theta^{\prime 2} y=f_{2}(x, y, z), \\
z^{\prime \prime}=f_{3}(x, y, z)
\end{gathered}
$$

where

$$
\begin{gathered}
f_{1}(x, y, z)=-v\left[\frac{1}{r_{13}^{3}}+\frac{3}{2 r_{13}^{5}} \epsilon\left[3 p_{x x 1}^{2}+p_{y y 1}^{2}+p_{z z 1}^{2}-\frac{5}{r_{13}^{2}}\left[\left(\frac{1-v}{u}+x\right)^{2} p_{x x 1}^{2}+y^{2} p_{y y 1}^{2}+z^{2} p_{z z 1}^{2}\right]\right]\right]\left(\frac{1-v}{u}+x\right)- \\
(1-v)\left[\frac{1}{r_{23}^{3}}+\frac{3}{2 r_{23}^{5}} \epsilon\left[3 p_{x x 2}^{2}+p_{y y 2}^{2}+p_{z z 2}^{2}-\frac{5}{r_{23}^{2}}\left[\left(-\frac{v}{u}+x\right)^{2} p_{x x 2}^{2}+y^{2} p_{y y 2}^{2}+z^{2} p_{z z 2}^{2}\right]\right]\right]\left(-\frac{v}{u}+x\right),
\end{gathered}
$$




$$
\begin{gathered}
f_{2}(x, y, z)=-v\left[\frac{1}{r_{13}^{3}}+\frac{3}{2 r_{13}^{5}} \epsilon\left[p_{x x 1}^{2}+3 p_{y y 1}^{2}+p_{z z 1}^{2}-\frac{5}{r_{13}^{2}}\left[\left(\frac{1-v}{u}+x\right)^{2} p_{x x 1}^{2}+y^{2} p_{y y 1}^{2}+z^{2} p_{z z 1}^{2}\right]\right]\right] y- \\
(1-v)\left[\frac{1}{r_{23}^{3}}+\frac{3}{2 r_{23}^{5}} \epsilon\left[p_{x x 2}^{2}+3 p_{y y 2}^{2}+p_{z z 2}^{2}-\frac{5}{r_{23}^{2}}\left[\left(-\frac{v}{u}+x\right)^{2} p_{x x 2}^{2}+y^{2} p_{y y 2}^{2}+z^{2} p_{z z 2}^{2}\right]\right]\right] y, \\
f_{3}(x, y, z)=-v\left[\frac{1}{r_{13}^{3}}+\frac{3}{2 r_{13}^{5}} \epsilon\left[p_{x x 1}^{2}+p_{y y 1}^{2}+3 p_{z z 1}^{2}-\frac{5}{r_{13}^{2}}\left[\left(\frac{1-v}{u}+x\right)^{2} p_{x x 1}^{2}+y^{2} p_{y y 1}^{2}+z^{2} p_{z z 1}^{2}\right]\right]\right] z- \\
(1-v)\left[\frac{1}{r_{23}^{3}}+\frac{3}{2 r_{23}^{5}} \epsilon\left[p_{x x 2}^{2}+p_{y y 2}^{2}+3 p_{z z 2}^{2}-\frac{5}{r_{23}^{2}}\left[\left(-\frac{v}{u}+x\right)^{2} p_{x x 2}^{2}+y^{2} p_{y y 2}^{2}+z^{2} p_{z z 2}^{2}\right]\right]\right] z,
\end{gathered}
$$

and $r_{13}^{2}=\left(\frac{1-v}{u}+x\right)^{2}+y^{2}+z^{2}$ and $r_{23}^{2}=\left(\frac{-v}{u}+x\right)^{2}+y^{2}+z^{2}$.

Equations 12 to 14 are given in a rotating reference frame, under the assumption that binary asteroid moves in a circular orbit, where the primaries are located on the $x$ axis.

\subsection{Lagrangian Points and Zero-Velocity Curves}

There are five Lagrangian points in the classical three-body problem with point masses. Three of them are collinear $\left(L_{1}, L_{2}, L_{3}\right)$ and two non-collinear $\left(L_{4}, L_{5}\right)$. The Lagrangian points $L_{4}$ and $L_{5}$, in the classical formulations, are located at equal distances from the primary bodies (Thornton \& Mariom 2004; Valtonen \& Karttunen 2006). This paper uses the classical approach to locate the equilibrium points in a full three body problem. The Lagrangian points are found by assuming that all of the forces acting on the system are in relative equilibrium.

The Lagrange equations are found just like in the classical method to locate the equilibrium points in a full three-body problem by letting $z=0, x^{\prime}=y^{\prime}=z^{\prime}=0, x^{\prime \prime}=y^{\prime \prime}=z^{\prime \prime}=0$ in equations 12 to 14 . This paper presents only the Lagrangian points that are close to the classical ones; the other points are ignored. To be brief, this paper omits a deep discussion about equilibrium points, but related references can be found in Woo (2014) and Woo \& Misra (2014).

The zero-velocity curves are found with the Jacobi constant. The equations of motion in equations 12 to 14 can be written as:

$$
\begin{gathered}
x^{\prime \prime}-2 \theta^{\prime} y^{\prime}=\frac{\partial \breve{U}}{\partial x}, \\
y^{\prime \prime}+2 \theta^{\prime} x^{\prime}=\frac{\partial \breve{U}}{\partial y}, \\
z^{\prime \prime}=\frac{\partial \breve{U}}{\partial z},
\end{gathered}
$$

where the gravitational potential $\breve{U}$ is given by (Woo, 2014):

$$
\begin{aligned}
\breve{U}(x, y, z)= & \frac{1}{2} \theta^{\prime 2}\left(x^{2}+y^{2}\right)+v\left[\frac{1}{r_{13}}+\epsilon \frac{3}{2 r_{13}^{3}}\left[\frac{P_{1}}{3}-\frac{\left(\frac{1-v}{u}+x\right)^{2} p_{x x 1}^{2}+y^{2} p_{y y 1}^{2}+z^{2} p_{z z 1}^{2}}{r_{13}^{2}}\right]\right]+ \\
& (1-v)\left[\frac{1}{r_{23}}+\epsilon \frac{3}{2 r_{23}^{3}}\left[\frac{P_{2}}{3}-\frac{\left(\frac{-v}{u}+x\right)^{2} p_{x x 2}^{2}+y^{2} p_{y y 2}^{2}+z^{2} p_{z z 2}^{2}}{r_{23}^{2}}\right]\right],
\end{aligned}
$$

where $P_{i}=p_{x x i}^{2}+p_{y y i}^{2}+p_{z z i}^{2}$ for $i=1,2$. 
The Jacobi constant can be re-written as:

$$
C\left(x, y, z, x^{\prime}, y^{\prime}, z^{\prime}\right)=2 \breve{U}(x, y, z)-\left(x^{2}+y^{\prime 2}+z^{\prime 2}\right) .
$$

Equation 22 can be re-arranged as a zero-velocity surface equation as follows:

$$
2 \breve{U}(x, y, z)-C\left(x, y, z, x^{\prime}, y^{\prime}, z^{\prime}\right)=\left(x^{\prime 2}+y^{\prime 2}+z^{\prime 2}\right)>0 .
$$

The Lagrangian points can be used to find critical values of the Jacobi constant $C$, e.g. $C_{L 1}=$ $C\left(x_{L 1}, 0,0,0,0,0\right)$ where $x_{L 1}$ is the position of the $L_{1}$ Lagrangian point.

\subsection{Search for Stable Orbits around the Binary System}

\subsubsection{Periodic Orbits}

The numerical search for periodic orbits, in this work, uses the same mathematical formulation as Roy (2005). The search for periodic orbits is conducted on the $X-Y$ plane, which rotates with velocity $\theta^{\prime}$ along with the OXYZ plane. It is considered that the third-body is on the $X$ axis and its velocity is on the perpendicular $Y$ axis, i.e., $x^{\prime}=z^{\prime}=y=z=0$ and $y^{\prime} \neq 0$.

Therefore, a periodic orbit in this case is the position $x_{\text {per }}$ that the satellite has when it crosses the $x$-axis at times $\tau_{1}\left(x_{p e r}\right), \tau_{2}\left(x_{p e r}\right), \tau_{3}\left(x_{p e r}\right), \ldots$, with the velocity $x^{\prime}$ assumed to be zero. The period of the orbit is given by $T=\tau_{i}\left(x_{p e r}\right)-\tau_{j}\left(x_{p e r}\right)$, for $i=j+1$ and $i \geq 2$ (Roy 2005).

The Jacobi constant is used for the search of periodic orbits. Substituting: $x^{\prime}=z^{\prime}=y=z=0 ; x=x_{p e r}$ and $y^{\prime}=y_{p e r}^{\prime} \neq 0$ in equation 23, we have (Roy 2005; Woo 2014):

$$
y^{\prime 2}=\theta^{\prime 2} x_{p e r}^{2}+2 v\left(\frac{1}{r_{13 p}}+\frac{\epsilon}{2 r_{13 p^{3}}}\left(P_{1}-3 p_{x x 1}^{2}\right)\right)+2(1-v)\left(\frac{1}{r_{23 p}}+\frac{\epsilon}{2 r_{23 p^{3}}}\left(P_{2}-3 p_{x x 2}^{2}\right)\right)-C,
$$

where $r_{13 p}=\left|\frac{1}{\frac{1-v}{u}+x_{p e r}}\right|$ and $r_{23 p}=\left|\frac{1}{\frac{-v}{u}+x_{p e r}}\right|$.

\subsubsection{The Grid Search Method}

The Grid Search Method (GSM) is used in this section to find stable orbits around the KW4 and Didymos binary systems.

The binary system will be modeled as irregular bodies, but for the grid search method it will be assumed that the primary asteroid is a point mass, to determine the initial state of the spacecraft.

The initial state of the spacecraft orbit is based on a circular Keplerian orbit around the primary body of the binary system; the second body is ignored in the computation. Therefore, the initial state of the spacecraft orbit is defined by the classical Keplerian elements: semi-major axis, eccentricity, inclination and true anomaly. The other two variables of the Keplerian elements are assumed to be zero. The center of the system used to compute the initial state of the spacecraft is the center of mass of the primary body. To compute the initial state of the spacecraft the mass of the system is considered to be the mass of the primary body. This estimation works well in this paper since the mass of the primary body is much larger than that of the secondary for both binary systems studied. Some adaptations should be done if the secondary body has a considerable mass compared to the primary body. The semi-major axis is normalized by dividing it by $L$. The inclination and true anomaly can be 0 or 180 degrees.

Numerical integrations map the orbits, and measure the lifetime of the orbits before a collision with one of the asteroids or an escape occurs. The GSM provides good data visualization of the regions where there are stable orbits. These maps were first shown in Oliveira \& Prado (2017).

The maximum simulation time if there is no escape or collision with one of the asteroids was arbitrarily chosen to be $\tau=42$. This period of time is assumed to be sufficient to estimate if the orbit is stable around the asteorids. The solar radiation pressure acts as a perturbation force that will always interfers with the trajectory of the satellite. Therefore, even if a closed periodic orbit is found around a binary system, the solar radiation pressure will eventually disturb the trajectory. 


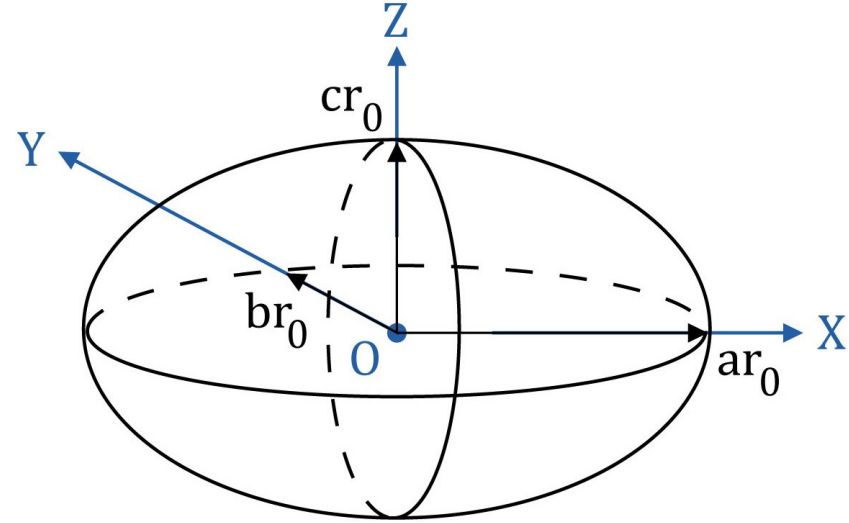

(a) Ellipsoid Shape.

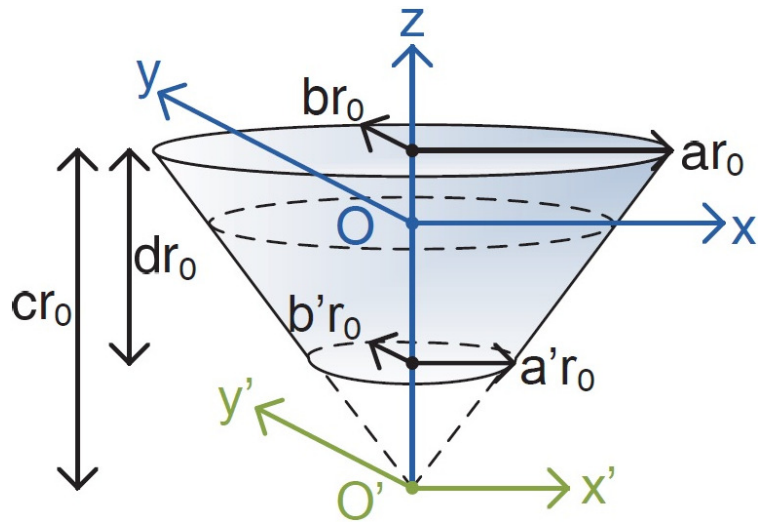

(b) Truncated Cone

Fig. 2. The shape of irregular bodies. The color figure can be viewed online.

\subsection{The Shape of the Asteroids}

The irregular shapes are shown in Figure 2 and the mathematical formulations of the gyration radii are given in Woo (2014) and Woo \& Misra (2013). The origin of the body-fixed $x_{i} y_{i} z_{i}$ frame is attached to the center of mass $O_{i}$, where $i=1$ or 2 . The $x_{i} y_{i} z_{i}$ are oriented along the principal axes. The dimensions of the ellipsoid are $a r_{0}, b r_{0}, c r_{0}$ in the $x_{i}, y_{i}, z_{i}$ directions, respectively.

For the truncated cone, at its largest base, the dimensions are $a r_{0}$ and $b r_{0}$ in the $x_{i}$ and $y_{i}$ directions, respectively. At the small base, the $x$ and $y$ dimensions are $a^{\prime} r_{0}$ and $b^{\prime} r_{0}$, respectively. The height of the full cone would be $c r_{0}$. The height of the truncated cone is $d r_{0}$. For ease of integration, a second $x^{\prime} y^{\prime} z^{\prime}$ frame is attached to the vertex $O^{\prime}$ of the full cone. The distance between $O$ and $O^{\prime}$ is obtained by computing the location of the center of mass $O$ in the $x^{\prime} y^{\prime} z^{\prime}$ frame.

\subsection{The Solar Radiation Pressure}

The solar radiation pressure is the pressure exerted upon any surface due to the exchange of momentum between the area of the surface and the momentum of light or electromagnetic radiation which is absorbed, reflected, or otherwise diffused.

The solar radiation force is non-conservative, and it can play a key role in spacecraft orbits in the Solar System. All spacecraft experience such a pressure, except when they are behind the shadow of a larger orbiting body.

In this work, the solar radiation flux is calculated based on the solar constant which is $1361 \mathrm{~W} / \mathrm{m}^{2}$ at 1 au (Kopp \& Lean 2011). The solar radiation flux depends on the distance between the spacecraft and the Sun.

The solar radiation pressure is not considered in the Lagrangian points and zero-velocity curves computation. All of the simulations consider the solar radiation pressure as a disturbing force in the dynamics of the spacecraft. The simulations start at the perigee of the center of mass of the binary system, where the solar radiation pressure has the largest influence on the spacecraft's dynamics. It is assumed that the spacecraft is a cube with one of the faces always facing the center of mass of the primary body. Each face is $10 \mathrm{~m}^{2}$; the mass of the spacecraft is considered to be $500 \mathrm{~kg}$.

The surface area element $d A$ is the elementary area of the spacecraft in $\mathrm{m}^{2}$. The constant $c$ is the speed of light. The solar flux is given by $\Psi\left(\mathrm{W} / \mathrm{m}^{2}\right)$.

The unit vector $\hat{\mathbf{s}}$ has the spacecraft-to-Sun direction. The unit vector $\hat{\mathbf{n}}$ is the surface area element unit normal vector. The angle $\phi$ is the angle between the surface normal and $\hat{\mathbf{s}}$. If $\cos (\phi)$ is negative, then the area element is not illuminated by the Sun and will not experience any radiation pressure. 
For each surface area element $d A$ of the spacecraft, the coefficients $\alpha, \rho$, and $\delta$ represent the fraction of the incident radiation that is absorbed, reflected, or diffused, respectively. These coefficients are related as follows:

$$
\alpha+\rho+\delta=1 .
$$

The total differential force due to solar radiation pressure on a surface area element $d \mathbf{F}$ is the sum of the differential forces of the absorbed $d \mathbf{F}_{\alpha}$, the speculary reflected $d \mathbf{F}_{\rho}$ and the diffuse $d \mathbf{F}_{\delta}$ differential forces, given by the following equation (Lyon 2004):

$$
d \mathbf{F}=d \mathbf{F}_{\alpha}+d \mathbf{F}_{\rho}+d \mathbf{F}_{\delta},
$$

where

$$
\begin{gathered}
d \mathbf{F}_{\alpha}=-\frac{\Psi d A \cos (\phi)}{c} \alpha \hat{\mathbf{s}}, \\
d \mathbf{F}_{\rho}=-\frac{\Psi d A \cos (\phi)}{c} 2 \cos (\theta) \rho \hat{\mathbf{n}}, \\
d \mathbf{F}_{\delta}=-\frac{\Psi d A \cos (\phi)}{c} \delta\left(\hat{\mathbf{s}}+\frac{2}{3} \hat{\mathbf{n}}\right) .
\end{gathered}
$$

The equation for the force due to radiation pressure acting on each flat plate is obtained by integrating equation 26 over the surface area, $A$, of each side of the spacraft's faces:

$$
\mathbf{F}=-\frac{\Psi A \cos \theta}{c}\left[(1-\rho) \hat{\mathbf{s}}+2\left[\frac{\delta}{3}+\rho \cos \theta\right] \hat{\mathbf{n}}\right] .
$$

After the non-dimensionalization of the variables given in $\S 2.1 .2$, the solar radiation pressure aceleration is added to the equations of motion of the spacecraft given in equations 12 to 14 .

\section{RESULTS}

\subsection{Physical Parameters for 1999 KW4 and Didymos}

Tables 1 and 2 present the physical parameters of the 1999 KW4 binary system and Tables 3 and 4 those of the Didymos binary system.

\subsection{Initial Parameters and Asteroid Shapes for 1999 KW4 and Didymos}

Tables 5 to 8 present the gyration radii and the initial parameters used in the simulations. The initial parameters for both binary asteorid system $u, \theta, \alpha_{1}, \alpha_{2}, u^{\prime}, \theta^{\prime}, \alpha_{1}^{\prime}$ and $\alpha_{2}^{\prime}$ were carefully chosen to satisfy a mutual circular orbit. The choice of $\alpha_{1}$ and $\alpha_{2}$, not being constants, must be included in equations (15) to (17). Due to lack of space, the equations were omitted here but can be found in Appendix A of Woo \& Misra (2014).

The primary asteroid of $1999 \mathrm{KW} 4$ was modeled as a double truncated cone, the secondary as an ellipsoid. The values of the parameters $a, b, c$ and $d$ for the asteroid shapes and also $r_{0}$ are based on the physical parameters given in Table 2.

Figures 3 and 4 present the distance between the asteroid bodies $R_{12}$ in meters and the rotation period of the primary $\left(\theta+\alpha_{1}\right)$ and secondary $\left(\theta+\alpha_{2}\right)$ bodies for 10 orbital periods for $1999 \mathrm{KW} 4$ and Didymos, respectively. The numerical simulations of Figures 3 and 4 are consistent with the numerical data for the binary system in Tables 1 to 4 . The bodies move in a nearly circular orbit and the rotation period of the asteroids is constant and stable. There are small variations of the distance between the bodies, but for practical purposes when the zero velocity curves are computed it is considered that the mutual asteroid orbits are circular.

For Didymos, the asteroid shape, dimensions and some of the initial parameters are estimated based on the current knowledge of its physical properties. The binary system is tidally locked (Landis \& Johnson 2019), therefore $\alpha_{2}^{\prime}=0$.

Current theories of asteroid satellite formation predict that the satellites should have similar or smaller densities than the primaries. From the system mass $5.27 \times 10^{11} \mathrm{~kg}$ and the diameter ratio Scheirich \& Pravec (2009), the calculated mass of the secondary is $5 \times 10^{9} \mathrm{~kg}$ (Cheng et al. 2016).

The primary and secondary Didymos bodies are shaped as ellipsoids and their dimensions are based on Table 4, and the mass estimation on Scheirich \& Pravec (2009); Cheng et al. (2016). 
TABLE 1

ORBITAL DATA FOR THE 1999 KW4 BINARY SYSTEM

\begin{tabular}{lcc}
\hline & Binary Asteroid System & \\
& $2548 \mathrm{~m}$ & Center of Mass around the Sun $^{2}$ \\
\hline Semi-major Axis & 0.0004 & $0.642291859 \mathrm{au}$ \\
Eccentricity & $17.4216 \mathrm{~h}$ & 0.68846023 \\
Orbital Period & & $0.514762413 \mathrm{yr}$ \\
\hline
\end{tabular}

${ }^{1}$ (Ostro et al. 2006). ${ }^{2}$ (Benner 2014).

TABLE 2

PHYSICAL PARAMETERS FOR $1999 \mathrm{KW}^{*}$

\begin{tabular}{lcc}
\hline & Primary Body & Secondary Body \\
\hline Mass & $2.253 \times 10^{12} \mathrm{~kg}$ & $2.488 \times 10^{11} \mathrm{~kg}$ \\
Dimensions & $1532 \times 1495 \times 1347 \mathrm{~m}$ & $571 \times 463 \times 349 \mathrm{~m}$ \\
Rotation Period & $2.7645 \mathrm{~h}$ & $17.4223 \mathrm{~h}$ \\
\hline
\end{tabular}

*(Ostro et al. 2006; Shi et al. 2017; Fahnestock \& Scheeres 2008).

TABLE 3

ORBITAL DATA FOR THE DIDYMOS SYSTEM*

\begin{tabular}{lcc}
\hline & Binary Asteroid System & Center of Mass around the Sun \\
\hline Semi-major Axis & $1180 \mathrm{~m}$ & $1.6445 \mathrm{au}$ \\
Eccentricity & 0.06 & 0.3836 \\
Orbital Period & $0.4958 \mathrm{~d}$ & $2.1087 \mathrm{yr}$ \\
\hline
\end{tabular}

*(Zhang et al. 2017; Dell'Elce et al. 2017).

TABLE 4

PHYSICAL PARAMETERS FOR THE DIDYMOS SYSTEM*

\begin{tabular}{ll}
\hline Primary diameter & $780 \mathrm{~m}$ \\
Secondary Diameter & $163 \mathrm{~m}$ \\
Total mass & $5.278 \times 10^{11} \mathrm{~kg}$ \\
Density $(\rho)$ & $2100 \mathrm{~kg} / \mathrm{m}^{3}$ \\
Primary rotation period & $2.26 \mathrm{~h}$ \\
\hline
\end{tabular}

*(Zhang et al. 2017; Dell'Elce et al. 2017).

TABLE 5

SHAPE AND GYRATION RADII FOR THE 1999 KW4 ASTEROID BODIES

\begin{tabular}{lccccccc}
\hline & $\mathrm{a}$ & $\mathrm{b}$ & $\mathrm{c}$ & $\mathrm{d}$ & $p_{x x}$ & $p_{y y}$ & $p_{z z}$ \\
\hline Double Truncated Cone & 1 & 0.9758 & 1.2 & 0.8792 & 0.4379 & 0.4462 & 0.5460 \\
Ellipsoid & 0.3727 & 0.3022 & 0.2278 & - & 0.1662 & 0.1953 & 0.2146 \\
\hline
\end{tabular}


TABLE 6

INITIAL PARAMETERS FOR 1999 KW4

\begin{tabular}{cccccccccccc}
\hline$u_{c}$ & $\theta_{c}$ & $\alpha_{1}$ & $\alpha_{2}$ & $u_{c}^{\prime}$ & $\theta_{c}^{\prime}$ & $\alpha_{1}^{\prime}$ & $\alpha_{2}^{\prime}$ & $v$ & $\varepsilon$ & $L$ & $r_{0}$ \\
\hline 1.0010 & 0 & 0 & 0 & 0 & 1.0112 & 5.3014 & 0.0012 & 0.9457 & 0.0904 & $2548 \mathrm{~m}$ & $766 \mathrm{~m}$ \\
\hline
\end{tabular}

TABLE 7

SHAPE AND GYRATION RADII FOR THE DIDYMOS ASTEROID BODIES

\begin{tabular}{lccccccc}
\hline & mass & $\mathrm{a}$ & $\mathrm{b}$ & $\mathrm{c}$ & $p_{x x}$ & $p_{y y}$ & $p_{z z}$ \\
\hline Primary Ellipsoid & $5.288 \times 10^{11}$ & 1 & 0.9500 & 0.8697 & 0.5760 & 0.5927 & 0.6168 \\
Secondary Ellipsoid & $5 \times 10^{9}$ & 0.2090 & 0.1990 & 0.1900 & 0.1230 & 0.1263 & 0.1291 \\
\hline
\end{tabular}

TABLE 8

INITIAL PARAMETERS FOR THE DIDYMOS ASTEROIDS

\begin{tabular}{cccccccccccc}
\hline$u_{c}$ & $\theta_{c}$ & $\alpha_{1}$ & $\alpha_{2}$ & $u_{c}^{\prime}$ & $\theta_{c}^{\prime}$ & $\alpha_{1}^{\prime}$ & $\alpha_{2}^{\prime}$ & $v$ & $\varepsilon$ & $L$ & $r_{0}$ \\
\hline 0.998 & 0 & 0 & 0 & 0 & 1.0028 & 6.2559 & 0 & 0.9905 & 0.1092 & $1180 \mathrm{~m}$ & $390 \mathrm{~m}$ \\
\hline
\end{tabular}

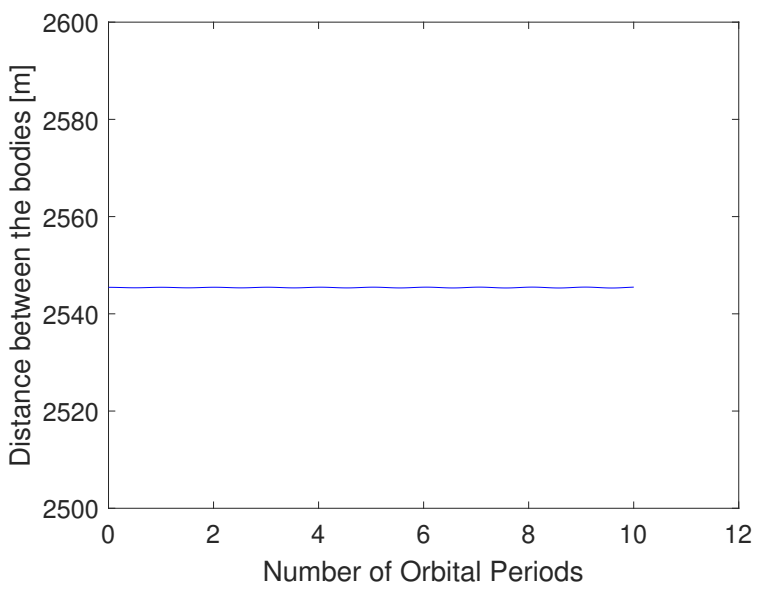

(a) Distance $R_{12}$.

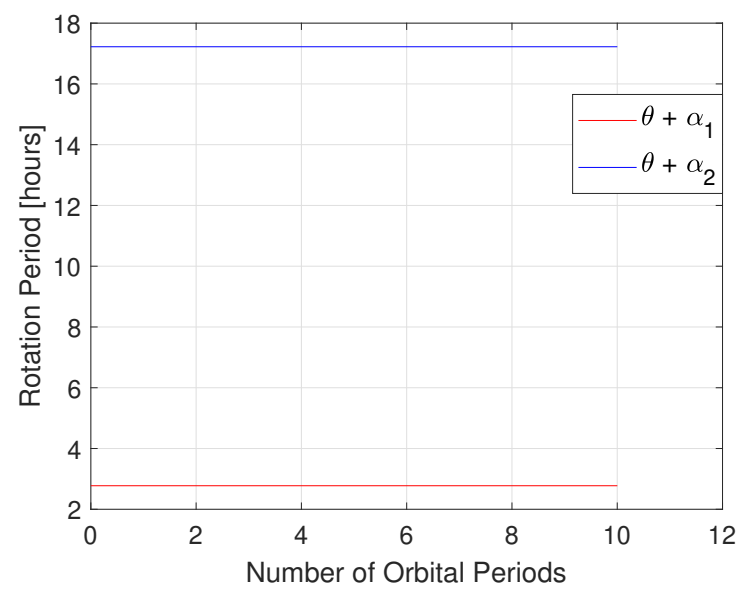

(b) Rotation Period.

Fig. 3. 1999 KW4 binary system simulation. The color figure can be viewed online.

\subsubsection{GSM application in the Binary Asteroid System}

This section presents the results of the GSM for the 1999 KW4 and Didymos binary systems. The GSM maps for 1999 KW4 and Didymos are very similar, therefore only the best GSM maps are shown for Didymos when the orbits are retrograde (Morais \& Giuppone 2012; Scheeres et al. 2017).

Figures 5a and 5b present the maps, based on the GSM, for the 1999 KW4 binary system when the inclination is zero and the true anomaly is 0 and $\pi$, respectively. The zero inclination results in prograde orbits.

Figures 6a and 6b present the maps, based on the GSM, for the 1999 KW4 binary system for retrograte orbits when the inclination is $\pi$ and the true anomaly is 0 and $\pi$, respectively. Figures $7 \mathrm{a}$ and $7 \mathrm{~b}$ show maps for the Didymos binary system when the inclination is $\pi$ and the true anomaly is 0 and $\pi$, respectively.

As expected, there are many more stable retrograte orbits than prograde orbits around the binary asteroid systems (Morais \& Giuppone 2012; Scheeres et al. 2017). The GSM is a good method to find stable orbits 


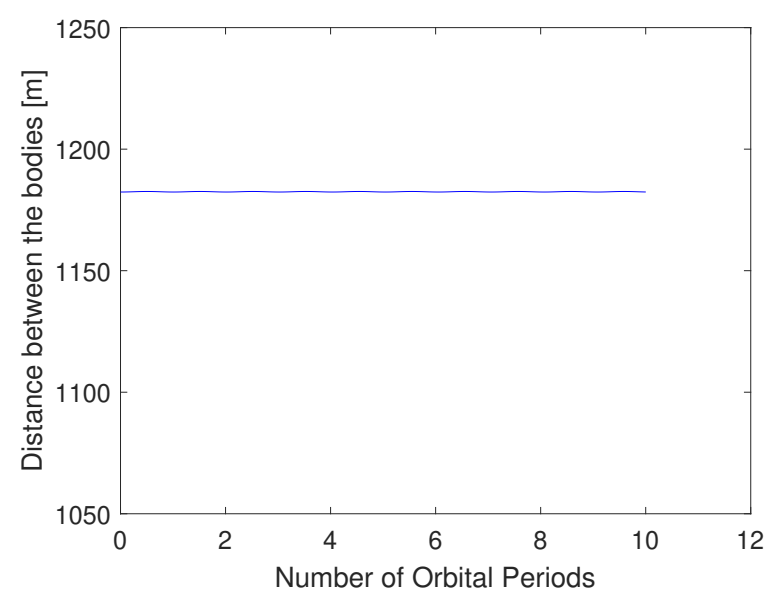

(a) Distance $R_{12}$

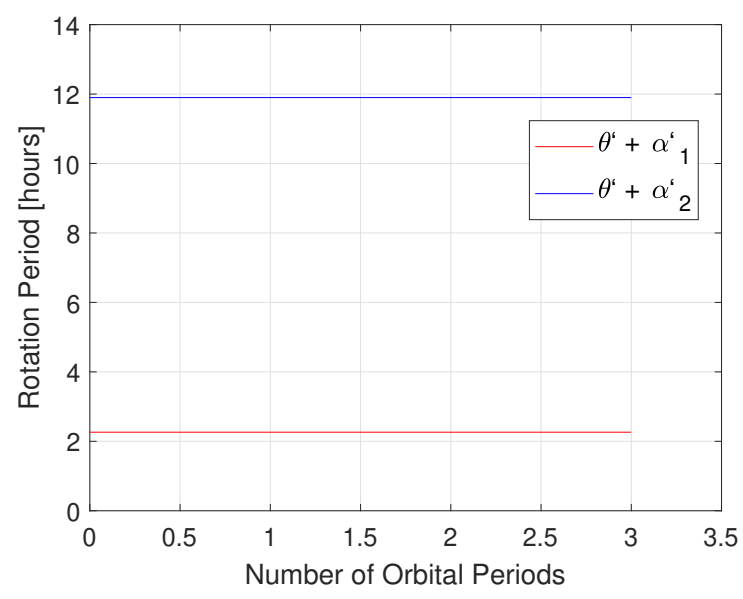

(b) Rotation Period

Fig. 4. Dydymos Binary System Simulation. The color figure can be viewed online.

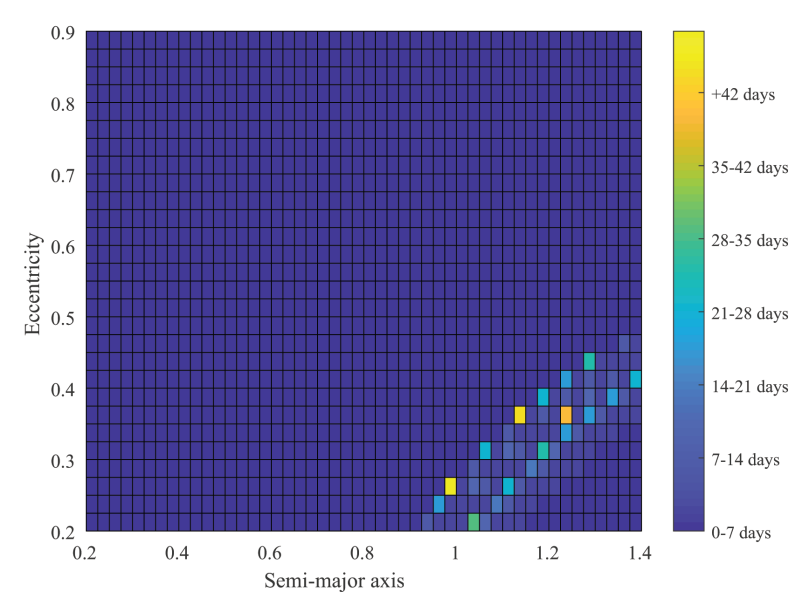

(a) True Anomaly $=0$

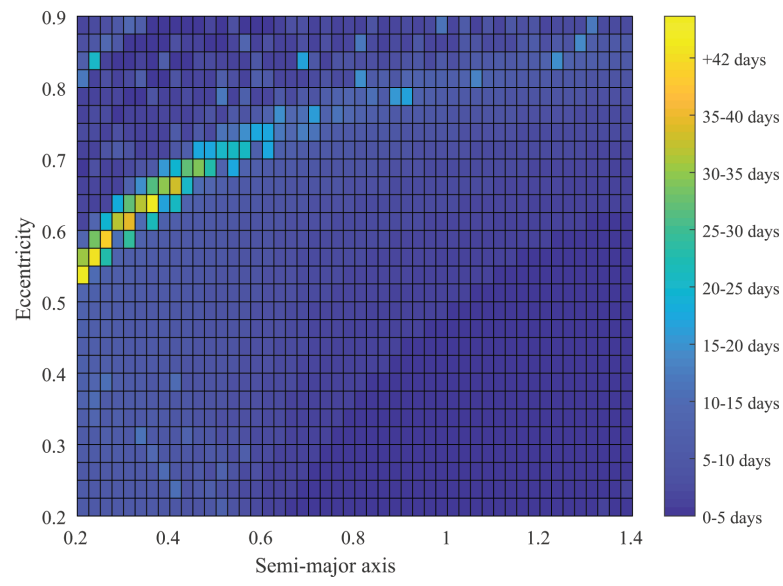

(b) True Anomaly $=\pi$

Fig. 5. Prograde orbits around 1999 KW4. The color figure can be viewed online.

around binary asteroid systems when the mass of the primary body is much bigger than that of the secondary. The secondary body acts as a perturbation on the Keplerian orbit, along with the non-spherical shape of the asteroid bodies and the solar radiation pressure. The inner orbits in the stable orbit band of Figure 6 are the best candidate orbits in which to place the spacecraft. These orbits are surrounded by stable orbits as well, therefore slight external disturbing forces such as the solar radiation pressure will not significantly change the stability.

Figure 8 shows examples of stable orbits around 1999 KW4 based on the orbits found by the GSM shown in Figure 6.

\subsection{Zero-Velocity Curves}

Zero-velocity curves can be used to find stable orbits confined to certain regions, e.g., an orbit confined around the primary body. 


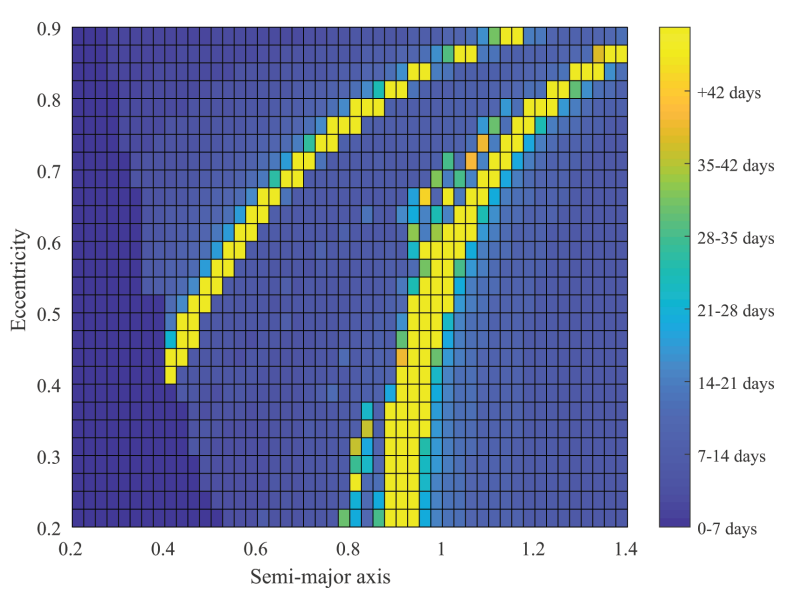

(a) True Anomaly $=0$

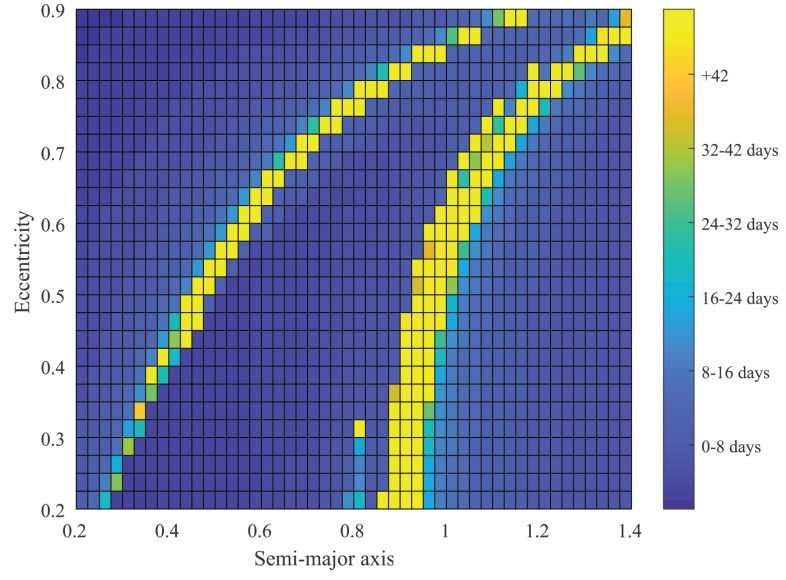

(b) True Anomaly $=\pi$

Fig. 6. Retrograte orbits around 1999 KW4. The color figure can be viewed online.

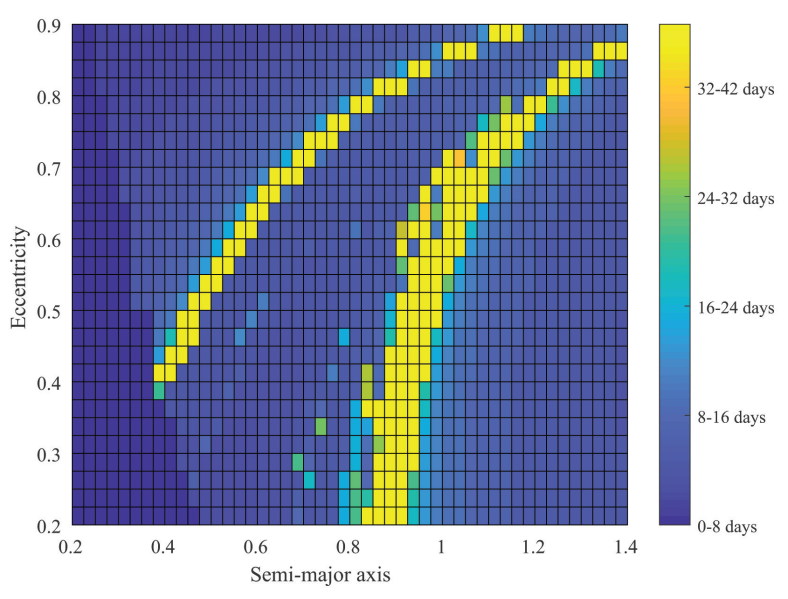

(a) True Anomaly $=0$

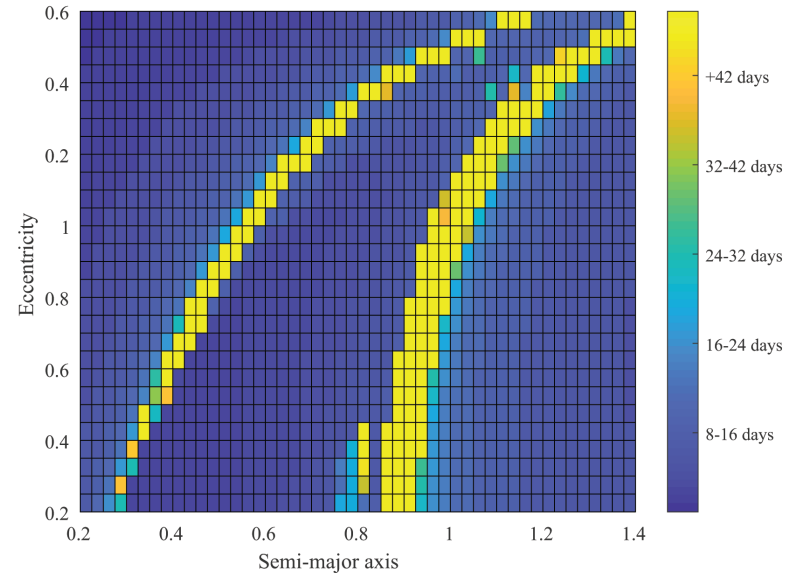

(b) True Anomaly $=\pi$

Fig. 7. Retrograte orbits around Didymos. The color figure can be viewed online.

Table 9 presents the Lagragian points and also the Jacobi constant $C$ based on these Lagrangian points computed for 1999 KW4 and Didymos, respectively. Figures 9 show the Jacobi constant $C$ of KW4 and Didymos based on the Lagrangian points $L_{1}, L_{2}$ and $L_{3}$, labelled $C_{L 1}, C_{L 2}$ and $C_{L 3}$, respectively.

As shown in Figure 9, for a spacecraft with a specific value of $C_{0}>C_{L 1}$, the zero-velocity surface consists of small ellipsoid-like geometries around the primary and secondary, and a large cylindrical-like surface surrounding the two-body system. The grey ellipsoidal shapes denote the asteroid bodies; the purple stars indicate the locations of the Lagrangian points. For $C_{L 1}>C_{0}>C_{L 2}$, the allowable region for the spacecraft is the two ellipsoidals connected around the asteroid bodies. And for $C_{L 2}>C_{0}>C_{L 3}$, the inner and outer allowable regions connect behind the secondary body.

Figure 10 presents orbits around the primary asteroid for planar motion, where $C_{0}=3.55$.

From a numerical search for the $1999 \mathrm{KW} 4$ system, it was found that $x(0)=-0.5$ produces a retrograde orbit around the primary body with $y(0)=z(0)=x^{\prime}(0)=z^{\prime}(0)=0$. From equation $24, y^{\prime}(0)=-1.0672$ (see Figure 10a). 


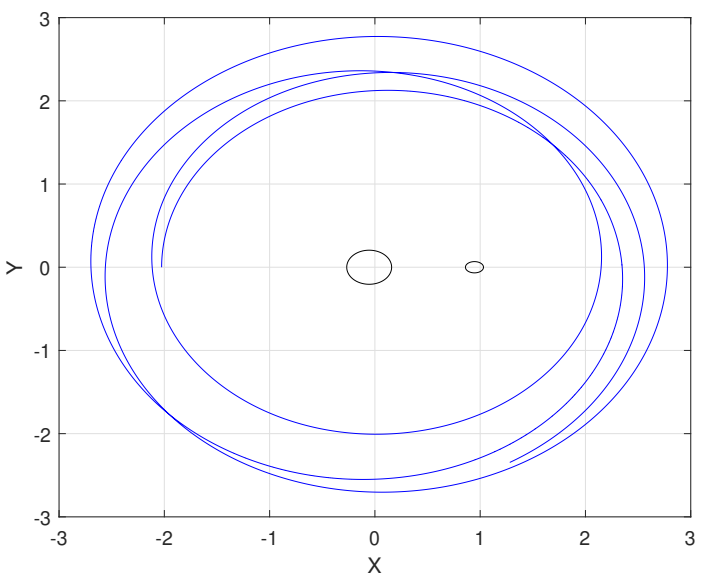

(a) Semi-major axis $=2.05$, eccentricity $=0$, true anomaly $=0$.

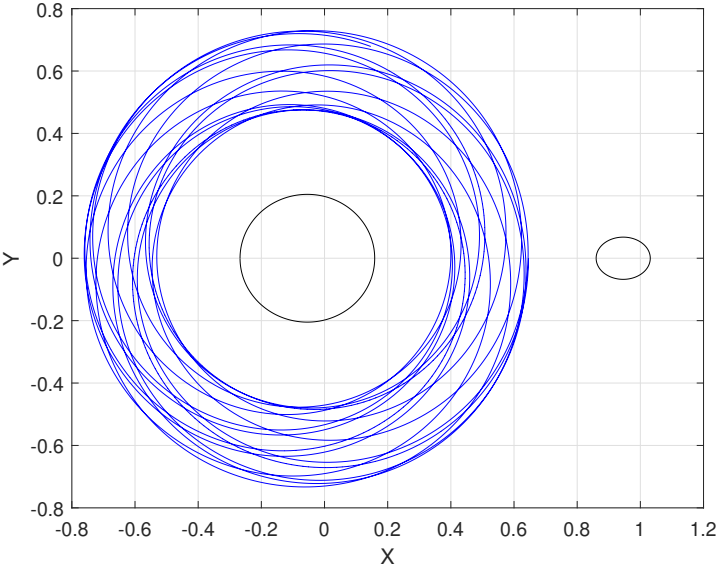

(b) Semi-major axis $=0.35$, eccentricity $=0.75$, true anomaly $=\pi$.

Fig. 8. Stable orbits around 1999 KW4. The color figure can be viewed online.

TABLE 9

\section{LAGRANGIAN POINTS}

\begin{tabular}{lcccccc}
\hline & & $L_{1}$ & $L_{2}$ & $L_{3}$ & $L_{4}$ & $L_{5}$ \\
\hline KW4 & $(x, y)$ & $(0.6792,0)$ & $(1.1953,0)$ & $(-0.9887,0)$ & $(0.4192,0.8897)$ & $(0.4192,-0.8897)$ \\
& $\mathrm{C}$ & 3.4812 & 3.3931 & 3.0701 & 2.9873 & 2.9873 \\
& & & & & & \\
\multirow{2}{*}{ Didymos } & $(x, y)$ & $(0.6907,0)$ & $(1.2098,0)$ & $(-1.0235,0)$ & $(0.4032,0.8972)$ & $(0.4032,-0.8972)$ \\
& $\mathrm{C}$ & 3.3725 & 3.1853 & 3.0229 & 2.9985 & 2.9985 \\
\hline
\end{tabular}

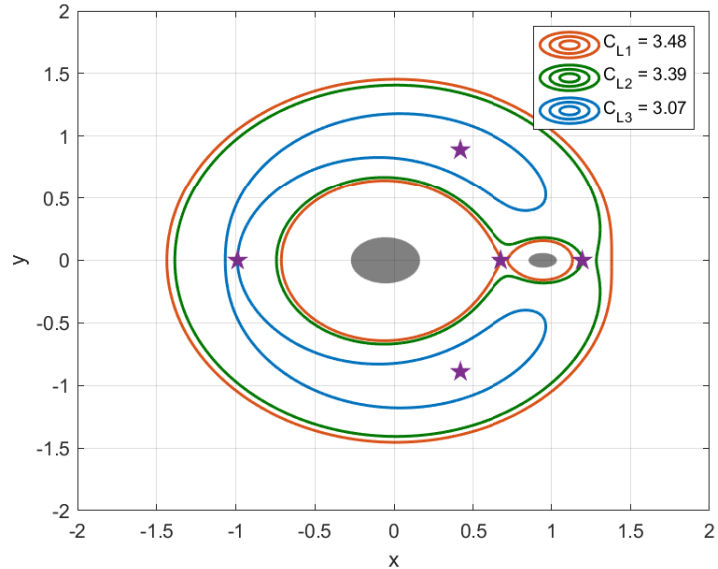

(a) $1999 \mathrm{KW} 4$

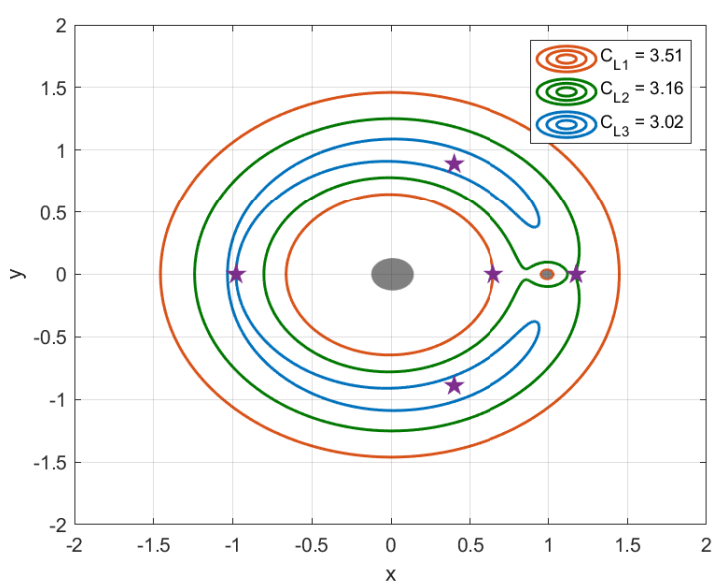

(b) Didymos

Fig. 9. Zero-velocity curves for $C_{1}, C_{2}$ and $C_{3}$. The color figure can be viewed online. 


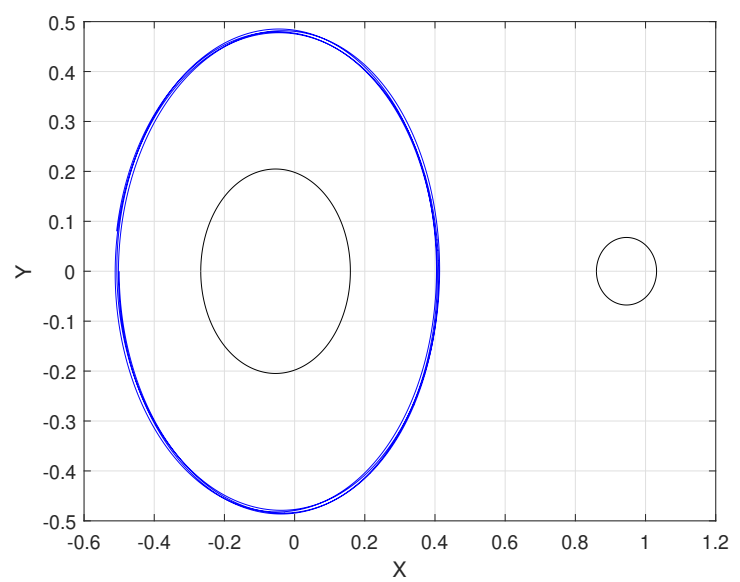

(a) 1999 KW4 binary system.

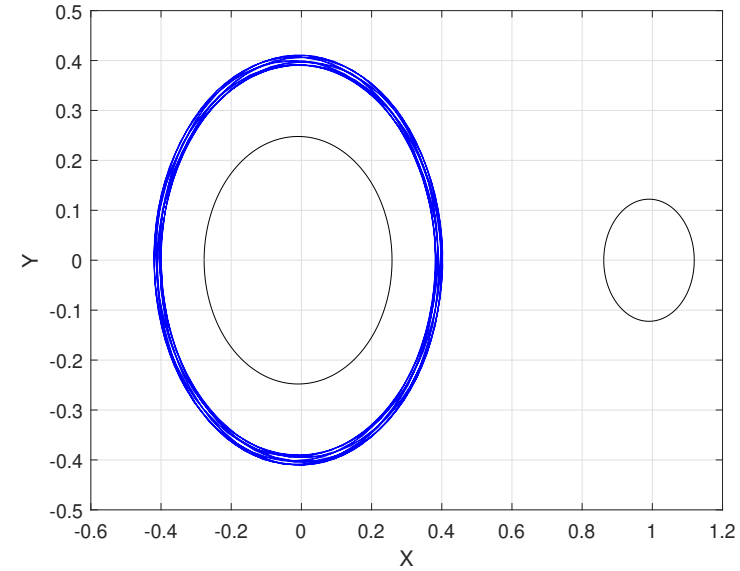

(b) Didymos binary system

Fig. 10. Trajectory of the spacecraft in the $X Y$-plane around the primary asteroid. The color figure can be viewed online.

For Didymos, a numercial search found a periodic retrograde orbit when $x(0)=-0.4$ and $y^{\prime}(0)=-1.2759$ (see Figure 10b).

Due to the high sensitivity on the initial conditions and to the solar radiation pressure perturbation, the resulting trajectories in Figure 10 are not perfectly periodic, but they may be suffient for practical purposes.

\section{CONCLUSION}

The binary asteroid systems are modeled as full irregular bodies in a restricted three-body problem. The physical and orbital parameters of binary asteroid systems 1999 KW4 and Didymos are considered in the mathematical formulation of the shape and dynamics of the system. Both binary asteroid systems have nearly circular mutual orbits; therefore, zero-velocity curves can also be used to find stable orbits around the system.

The paper proposes two distinct methods to search for stable orbits around the binary asteroids. The first method, named the grid search method, results in easy and practical visualization of stable orbits around the binary system. The maps are based on the initial state of the spacecraft and the time period of the spacecraft orbit before a collision or an escape occurs.

The second method to look for stable orbits is based on zero-velocity curves. The method has proved to be efficient to find stable orbits around different allowable regions based on the Jacobi constant.

The greatest advantage of the methods studied in this paper is to easily find stable orbits around binary asteroid systems in a fast and practical way using the grid zearch method and/or zero-velocity curves based on the full restricted circular three-body problem.

The authors gratefully acknowledge the financial support of FAPESP (2016/01430-7).

\section{REFERENCES}

Bellerose, J. \& Scheeres, D. J. 2008, AcAau, 62, 563, https://doi.org/10.1016/j.actaastro.2008.01.018

Bellerose, J. \& Scheeres, D. J. 2008, JGCD, 31, 1, https: //doi.org/10.2514/1.30937

Benner, L. A. M. 2014, Binary and ternary near-Earth asteroids detected by radar, JPL, website: http://echo. jpl.nasa.gov/ lance/binary.neas.html, accessed March 26,2019
Chappaz, L. \& Howell, K. C. 2015, CeMDA, 123, 123, https://doi.org/10.1007/s10569-015-9632-5

Cheng, A. F., et al. 2016, AcAau, 115, 262, doi: 10.1016/j.pss.2015.12.004

Dell'Elce, L., Baresi, N., Naidu, S. P., Benner, L. A. M., \& Scheeres, D. J. 2017 AdSpR, 59, 1304, https: //doi.org/10.1016/j.asr.2016.12.018

Fahnestock, E. G. \& Scheeres, D. J. 2008, Icar, 194, 410 
Hou, X. \& Xin, X. 2018, AsDyn, 2, 39, https://doi.org/10. 1007/s42064-017-0010-9

Hu, W. \& Scheeres, D. J. 2004, P\&SS, 52, 685, doi: 10.1016/j.pss.2004.01.003

Johnston, W. R. 2014, PDSS, 219

JPL, 2019, Center for Near Earth Object Studies, https:// cneos.jpl.nasa.gov/stats/totals.html (2019), Accessed 01 May 2019

JPL, 2019, Goldstone Radar Observations Planning: Asteroid 1999 KW4 in 2019, https://echo.jpl.nasa.gov/ asteroids/1999KW4/1999KW4_planning.2019.html (2019), Accessed 15 May 2019

Kopp, G. \& Lean, J. L. 2011, GeoRL, 38, 1706, doi: 10.1029/2010GL045777

Landis, R. \& Johnson, L. 2019, AcAau, 156, 394, https: //doi.org/10.1016/j.actaastro.2018.06.020

Lyon, R. H. 2004, Geosynchronous Orbit Determination Using Space Surveillance Network Observations and Improved Radiative Force Modeling, Mater of Science, Department of Aeronautics and Astronautics, 2004

Margot, J.-L., Pravec, P., Taylor, P., Carry, B., Jacobson, S. 2015, in Asteroids IV, ed. P. M. F. DeMeo and W. F. Bottke (Tucson, AZ: University of Arizona Press), 355

Morais M. H. M., Giuppone C. A. 2012, MNRAS, 424, 52, https://doi.org/10.1111/j.1365-2966.2012.21151.x

NASA, 2019, Science: Solar System Exploration, https:// solarsystem.nasa.gov/asteroids-comets-and-meteors/ asteroids/in-depth (2019), Accessed 25 Abril 2019

Oliveira, T. C. \& Prado, A. F. B. 2017, AdAns, 160, 17

Ostro, S. J., et al. 2006, Sci, 314, 1276

Roy, A. E. 2005, Orbital Motion (4th ed.; Bristol UK: Institue of Physics Publishing)

Scheirich, P. \& Pravec, P. 2009, Icar, 200, 531

Scheeres, D. J., Ostro, S. J., Hudson, R. S., \& Werner, R. A. 1996, Icar, 121, 67, https://doi.org/10.1006/icar. 1996.0072
Scheeres, D. J., Williams B. G., \& Miller, J. K. 2000, JGCD, 23, 466, https://doi.org/10.2514/2.4552

Scheeres, D. J., Van Wal, S., Olikara, Z., \& Baresi, N. 2017, in: International Symposium on Space Technology and Science, Ehime, Japan, 3-9

Shang, H., Wu, X., \& Cui, P. 2015, Ap\&SS, 355, 69, https://doi.org/10.1007/s10509-014-2154-X

Shi, Y., Wang, Y., \& Xu, S. 2017, CeMDA, 129, 307, doi: 10.1007/s10569-017-9776-6.

. 2018a, AcAau, 163, 11, https://doi.org/10. 1016/j.actaastro.2018.10.014

2018b, CeMDA, 130, 32, https://doi.org/10. 1007/s10569-018-9827-7

Thornton, S. T. \& Marion, J. B. 2004, Classical Dynamics of Particles and Systems, (5th ed.; Thomson Brooks/Cole)

Valtonen, M. \& Karttunen, H. 2006, The Three-Body Problem, (Cambridge, MA: CUP)

Woo, P. 2014, Dynamics of a Spacecraft in the Vicinity of a Binary Asteroid System, Thesis, McGill University

Woo, P., Misra, A. K., \& Keshmiri, M. 2013, CeMDA, 117, 263, https://doi.org/10.1007/s10569-013-9512-9

Woo, P. \& Misra, A. K. 2014, AcAau, 99, 158, https: //doi.org/10.1016/j.actaastro.2014.02.019 AcAau, 110, 313, https://doi.org/10.1016/j. actaastro.2014.11.001

Werner, R. A. \& Scheeres, D. J. 1996, CeMDA, 65, 313, https://doi.org/10.1007/BF00053511

Yu, Y. \& Baoyin, H. 2012a, AJ, 143, 62, doi: 10.1088/0004$6256 / 143 / 3 / 62$

. 2012b, MNRAS, 427, 872, doi: 10.1111/j.13652966.2012.21963.x

Zamaro, M. \& Biggs, J. 2015, CeMDA, 122, 263, https: //doi.org/10.1007/s10569-015-9619-2

Zhang, Y., et al. 2017, Icar, 294, 98, https://doi.org/10. 1016/j.icarus.2017.04.027

Thais C. Oliveira and Antonio F. B. A. Prado: Space Mechanic and Tecnology, National Institute for Space Research/INPE, Av. dos Astronautas 1758, Sao Jose dos Camopo, SP, Brazil, 12227-010 (thais.oliveira, antonio.prado@inpe.br). 